\title{
Immigrant's Vote? A Postponed Challenge for Albania
}

\author{
PhD. Afrim Krasniqi \\ University Aleksandër Moisiu, Durrës \\ E-mail: afrimkrasniqi@gmail.com
}

\section{Doi:10.5901/mjss.2014.v5n7p498}

\begin{abstract}
Even though Albania is one of the smallest European countries, it has one of the highest rates of emigration. It is among the few countries that have not yet implemented the participation of emigrants abroad in political elections. The study analyzes the problems of the case of Albania, the features of emigration and the political behavior with the regard to it, the constitutional and legal rapports, as well as the contrast between the international commitments of Albania, the expectations of emigrants themselves, the electoral promises of the parties, and the fragile practical achievements. My paper argues that due to the significant importance that emigration has with regard to the total number of population, the regional and global trend of granting emigrants the voting right, and the need of the political system for expansion, competition, inflows of ideas and energy, Albania has to make an immediate, stable and effective choice for involving all emigrants in political decision making.
\end{abstract}

\section{Introduzione}

Il dibattito sul voto degli emigranti (cittadini albanesi che vivono all'estero) nelle elezioni politiche è un dibattito nuovo, di tendenza e costituirà uno dei temi più roventi nei prossimi anni. L'Albania dispone di un grande potenziale migratorio e, a differenza dagli altri paesi della regione, dalla modalità in cui verrà risolta detta questione dipenderà anche il nuovo modello del sistema politico e governativo del paese. Quanto al numero dei voti gli emigranti albanesi hanno la potenzialità di influenzare su qualsiasi deliberazione politica importante, compresa l'elezione della maggioranza del governo oppure l'elezione del Presidente (nel caso del passaggio alle elezioni dirette).

Come succede anche per gli altri aspetti dei concetti e delle istituzioni democratiche anche il dibattito sul voto dell'emigrazione giunge in ritardo in Albania rispetto agli altri paesi, compresi i nostri vicini, dove questo voto ormai viene applicato. II ritardo nel trattare e risolvere questo problema riflette il grande peso politico e le altre peculiarità del caso albanese in rapporto agli analoghi modelli regionali ed europei.

\section{La Costituzione ed il Voto dell'emigrazione}

L'Albania ha sottoscritto la Dichiarazione Generale sui Diritti dell'Uomo, il cui art. 21 scrive espressamente «chiunque ha il diritto di partecipare al governo del suo paese, in modo diretto oppure tramite i rappresentanti scelti liberamente», un principio questo che comprende anche il diritto dei cittadini dentro e fuori i confini di partecipare al governo del loro paese. Nell'ambito interno costituzionale, l'Albania ha ormai una Costituzione approvata con il referendum del 1998. II diritto al voto dei cittadini è un principio costituzionale e fa parte delle libertà politiche del cittadino. Nella Costituzione Albanese, art. 45, si prevede che «ogni cittadino che ha compiuto 18 anni, anche il giorno stesso delle elezioni, ha il diritto di votare ed essere votato». La Costituzione riconosce il principio che «il voto è personale, uguale, libero e segreto» ed esclude dal diritto del voto soltanto i cittadini che, con una sentenza di tribunale passata in giudicato, sono stati dichiarati non capaci di intendere e volere. Gli stessi principi vengono sanciti anche dal Codice Elettorale della Repubblica d'Albania (2008, art. 3).

Così appare che la Costituzione si faccia garante del voto di ogni cittadino, in pratica però, a differenza degli altri modelli costituzionali di alcuni paesi europei, la Costituzione Albanese non ha una definizione precisa per il cittadino che vive all'estero. II concetto degli albanesi "all'estero" nella Costituzione è collegato agli albanesi del Kosovo, della Macedonia ed altri paesi, dove gli albanesi sono cittadini. L'art. 8 della Costituzione riconosce allo stato albanese la responsabilità di assicurare «sostegno ai cittadini albanesi che vivono e lavorano all'estero per conservare e sviluppare $i$ legami con la tradizione culturale nazionale». Anche la legislazione seguente sui diritti dell'uomo e sulle elezioni non fa una distinzione tra i cittadini «dentro» ed i cittadini «fuori», oppure tra i cittadini all'estero che sono residenti temporanei e quelli che sono residenti di lungo periodo. L'unica definizione legale particolare è quella fatta nella legge albanese 
sull'emigrazione, secondo la quale, viene sancito l'obbligo dello stato a creare facilitazioni «agli emigranti a rientrare nel paese per esercitare il diritto al voto a seconda del Codice Elettorale» (FZ [Quaderno Ufficiale], 2007).

L'Albania ha accettato come standard di riferimento le valutazioni della Commissione di Venezia e del Consiglio d'Europa. Di conseguenza, nel caso del voto degli emigranti l'Albania accetta ufficialmente la definizione del Consiglio d'Europa che «la votazione all'estero garantisce l'equità tra i cittadini che vivono nel paese e gli emigranti. Essa assicura che i cittadini conservino i legami con il loro paese d'origine e accresce i loro sentimenti di appartenenza alla nazione, dove sono membri indipendentemente dalle circostanze geografiche, economiche e politiche» (Consiglio d'Europa, 2011). Comunque, nell'analisi pratica c'è un divario tra l'accettazione del concetto e l'azione concreta nella sua applicazione.

\section{La Struttura Politica della Diaspora Albanese nel Mondo}

Secondo l'Istituto delle Statistiche (INSTAT) la popolazione albanese conta circa 3.4 milioni di abitanti, di cui un quarto vive un'emigrazione attiva. La parte dell'emigrazione costituisce circa il $35 \%$ della popolazione attiva e i flussi migratori albanesi sono stati 5-6 volte superiori a quelli dei paesi in via di sviluppo, in rapporto alla popolazione attiva. INSTAT rileva che il $75 \%$ di loro sono maschi ed il $25 \%$ sono femmine, rapporto che conferma anche il dominio generazionale nella manodopera. (INSTAT, 2002). II numero più rilevante degli emigranti nel mondo dal censimento fatto risulta essere in Grecia 600 mila albanesi, seguito dall'Italia con circa 420 mila, Stati Uniti 150 mila, Regno Unito 50 mila, e numeri più esigui in Germania, Canada, Belgio, Turchia, Francia, Austria, Svizzera, Paesi Bassi ecc. (SKM, 2004 e Caritas Immigrazione 2008).

Facendo riferimento agli studi storici l'Albania fa parte nella lista dei paesi caratterizzati da grandi flussi di emigrazione. Nella prima emigrazione (prima e dopo la Seconda Guerra Mondiale 1911-1916, tra le due Guerre (19241939), nel periodo dell'occupazione (1939-1944) e anche nel periodo dell'installazione del regime comunista (1944-1945) abbiamo non solo l'emigrazione economica, ma specialmente quella politica. Gli avversari di ogni regime e periodo sono stati costretti ad emigrare, influenzando in maniera rilevante la qualità della elite politica e culturale rimasta nel paese.

Dopo la caduta del sistema comunista il paese si è confrontato con una fuga massiva verso la Grecia e l'Italia (1991), nonché più tardi con fughe minori verso gli altri paesi, specialmente quelli occidentali. Un altro caso unico viene costituito dal 1997 con i tumulti politici in Albania e la ripresa della fuga massiva in particolar modo dalle regioni coinvolte nei tafferugli. Dopo gli anni 2000 ci sono casi e forme sporadiche di emigrazione, specialmente per motivi economici 0 unioni familiari. Negli ultimi anni gli indici dell'emigrazione sono minimi in confronto a qualsiasi periodo precedente. Una caratteristica comune di tutte le emigrazioni è l'emigrazione della nuova generazione, dei gruppi politici avversari, nonché il modello dell'emigrante economico.

Secondo le statistiche ufficiali del Ministero del Lavoro e delle Questioni Sociali gli albanesi con un totale di 0.8 milioni di migranti «costituiscono il terzo gruppo dei cittadini dei paesi terzi nell'UE» (MPÇS, 2011). Tenendo conto anche del fatto che esiste anche un'altra parte di cittadini albanesi che hanno emigrato con identità falsa, principalmente dalle zone albanesi dell'ex Jugoslavia.

\section{Da Emigranti in Elettori Potenziali}

Nella prima fase della transizione (1991-2001) nessun partito politico albanese non si era focalizzato con il proprio programma sul voto degli emigranti. I programmi elettorali includevano la cura dei loro diritti nei rispettivi paesi, sostegno nelle questioni d'istruzione e culturali, la cura per le politiche sociali ed il contributo degli anni di lavoro, nonché altri aspetti universali. In questa fase la stessa emigrazione non aveva un'organizzazione regolare politica e rappresentava una stratificazione complessa, senza identità e senza chiare priorità oltre al bisogno d'integrazione e di migliori condizioni di vita.

Dopo l'anno 2002 gli esperti internazionali di IOM (Organizzazione Internazionale per la Migrazione) hanno aiutato il governo albanese nella stesura della Strategia Nazionale per la Migrazione, nonché nella creazione di un database modesta per gli albanesi al mondo. II progetto culminò con l'approvazione nel 2004 della Strategia, nella quale, per la prima volta, viene ribadita la necessità dell'inclusione della migrazione nelle elezioni politiche in Albania (SKM, 71). La strategia del governo e dell'IOM prevedeva che il diritto del voto si applicasse in Albania nelle elezioni dell'anno 2009, cioè dopo 5 anni di preparativi. In effetti, i partiti politici al governo e in opposizione sono intervenuti per più volte al Codice Elettorale per apportare delle modifiche di breve termine $(2003,2006,2007,2008)$, ma in nessuno dei casi i principali partiti politici non hanno incluso nel dibattito e nelle modifiche il voto promesso all'emigrazione.

Nel 2008 un piccolo partito politico, nonché rete delle organizzazioni civili ha proposto la regolazione legale del 
voto degli emigranti prevedendo il voto nelle ambasciate, consolati, nonché per posta, però i due più grandi partiti politici (II Partito Democratico al potere ed il Partito Socialista in opposizione) non hanno accolto la proposta. I partiti hanno promesso di rivedere la loro posizione nella nuova riforma elettorale, dell'anno 2012. Anche in questo caso il Codice Elettorale ha apportato modifiche, ma in nessuna fase del dibattito e dei cambiamenti non è stata inclusa la questione del voto dell'emigrazione.

Dopo l'anno 2005 e specialmente negli anni 2011-2013 sono stati eseguiti un numero di appelli, petizioni e iniziative politiche, civili 0 delle varie comunità degli albanesi al mondo rivolti alle autorità albanesi richiedendo l'approvazione di un quadro legale per il voto garantito agli emigranti. La maggior parte delle iniziative sono venute dall'Italia, dalla Gran Bretagna, dalla Germania e dagli altri paesi occidentali, nei quali il diritto del voto della diaspora ha una lunga e felice tradizione. Tali iniziative sono intraprese anche da parte del terzo partito politico, II Movimento Socialista per l'Integrazione (LSI) nonché il gruppo giovanile G99, divenuto poi nel 2009 un partito politico e soggetto elettorale. Solo alcuni giorni prima delle elezioni parlamentari del 2013 il governo ha cambiato atteggiamento e ha intrapreso l'iniziativa politica per il diritto del voto degli emigranti. II suo movimento è stato interpretato come uno degli scenari tattici possibili per attirare voti di emigrazione o per vedere un'opportunità per un terzo mandato governativo, ma la sua offerta speculativa è stata rifiutata dall'opposizione.

\section{II Paradosso: Rifiuto del Voto ed i Partiti si Rivolgono agli Emigranti}

Nelle condizioni della diffusione delle critiche e delle diverse iniziative politiche per la regolamentazione legale del voto degli emigranti albanesi che vivono e lavorano in altri paesi, i principali partiti politici si sono incamminati in una via parallela: da una parte loro hanno trovato il consenso per il rifiuto di ogni iniziativa riguardo al voto dell'emigrazione, dall'altra parte ciascuno di loro ha cominciato a istituire filiali e strutture partitiche nei grandi gruppi della diaspora. Anche se la legge albanese non consente l'istituzione e l'attività dei partiti fuori i confini ufficiali della Repubblica d'Albania, i tre grandi partiti politici (PS, PD, LSI) hanno istituito filiali regolari in Italia, Grecia, Germania, Belgio e Stati Uniti. Ciascuno dei partiti ha apportato modifiche nei propri statuti facendo possibile che i capi delle filiali in emigrazione avessero posizioni pari ai capi delle filiali locali dentro il paese. Ognuno dei partiti ha creato dei gruppi politici che hanno intrapreso tour continui di incontri con albanesi dell'emigrazione, cercando di acquisire il loro sostegno politico e finanziario.

La prima prova del coinvolgimento evidente degli emigranti nella vita politica albanese risale alle elezioni locali del 2011. I due partiti politici hanno condotto una gara molto forte a Tirana, la capitale del paese, dove la differenza tra le parti concorrenti era di soli 10 voti per un totale di mezzo milione di elettori attivi. Proprio in queste elezioni ciascuna parte ha cercato ed è riuscita a portare dall'estero gruppi di emigranti sostenitori, il cui voto è stato decisivo nel risultato finale delle elezioni. I mass media hanno reso noto diverse volte che i partiti hanno pagato le spese di viaggio degli emigranti, mettendo a loro disposizione voli aerei oppure pullman (Italia e Grecia), fatto che non è stato smentito mai da nessuna delle parti anche se nessuna di loro non ha incluso questa spesa nelle spese elettorali presso la Commissione Centrale delle Elezioni (KQZ).

II ruolo attivo degli elettori emigranti il 2011 ha esortato i partiti a prepararsi ancora di più per le elezioni parlamentari del 2013. In queste elezioni è stato segnato un numero record di elettori emigranti organizzati dai partiti politici e venuti a votare dai paesi vicini, specialmente dalla Grecia e dall'Italia. Tali organizzazioni sono state fatte dai grandi partiti politici (PS, PD, LSI), ma anche dal principale partito di minoranza greca in Albania, PBDNJ.

II capo della filiale greca del Partito Socialista Albanese, A. Qirici ha dichiarato alle medie albanesi che nelle elezioni parlamentari del 2013 sono venuti circa 70 mila elettori emigranti per la coalizione della sinistra solo dalla Grecia (Shqip, 2013). Con la loro presenza ed il loro voto sono riusciti ad assicurare 6-7 mandati parlamentari. PS ha incluso nell'elenco dei candidati a Korça un rappresentante dell'emigrazione in Grecia. Le stesse fonti hanno ribadito che la coalizione sinistra è riuscita ad assicurare circa 120 mila voti dagli emigranti arrivati, 11-12 deputati. Lo stesso calcolo vale anche per i flussi migratori per i voti alla destra e di conseguenza, per un incremento rilevante della presenza e del voto raccomandato degli emigranti nel parlamento albanese.

\section{Modelli di Suffragio degli Emigranti in Regione}

La Commissione di Venezia ed il Consiglio Europeo (2010) elencano l'Albania nella lista dei 12 stati nei quali non sono stati intrapresi atti legali preliminari per rendere possibile la votazione dei cittadini che vivono all'estero. Nella lista fanno parte anche altri paesi della regione quali Turchia, paese il quale nel 2013 ha modificato la legge rendendo possibile il voto della diaspora per le elezioni presidenziali. In quest'analisi l'Albania fa parte della lista dei quattro stati (assieme a Malta, Cipro e Andorra) che non hanno sancito la votazione della diaspora nella costituzione e neanche nella legislazione 
elettorale. I cittadini possono venire nel loro paese di origine, votare ma anche in questo caso l'Albania differisce p.es. dalla Malta, stato nel quale agli elettori arrivati viene rimborsato il $90 \%$ delle spese del loro viaggio.

Un numero di paesi compresi i paesi vicini all'Albania, la Bulgaria, la Croazia, l'Italia, la Romania, la Serbia, la Slovenia ecc. hanno sancito per legge il diritto del voto ai cittadini dei loro paesi che vivono all'estero con residenza permanente o provvisoria. Ci sono altri paesi quali la Bosnia o la Macedonia dove i cittadini residenti provvisori all'estero fanno parte delle liste ufficiali degli elettori. La stessa pratica sceglie anche l'Albania. In ogni elezione la lista degli elettori è molto più lunga rispetto al numero degli elettori, poiché nella prima lista vengono inclusi cittadini a seconda del censimento generale, indipendentemente dalla loro residenza temporanea all'estero. Ci sono paesi quali la Germania dove vengono applicati standard e diritti diversi tra i cittadini residenti temporanei e quelli residenti permanenti all'estero.

La votazione dall'estero costituisce una pratica di votazione complicata e difficile. Problemi emergono nella registrazione degli elettori (pratica dell'inclusione nella lista e quella del termine di registrazione degli stessi elettori). In paesi quali Kosovo e Macedonia gli elettori si registrano negli uffici diplomatici o consolari, pratica che viene applicata anche in altri paesi della regione quali l'Italia e Serbia, nonché in almeno 15 altri paesi europei. C'è anche una lista di pochi paesi quali Bosnia, Bulgaria ecc. dove gli elettori devono essere registrati presso le autorità locali dentro i confini del paese (KE, 2011).

Le modalità di votazione dei cittadini all'estero sono molteplici. Si pratica il voto individuale, il voto preliminare 0 il voto elettronico. In un numero di paesi, compresa la Croazia, la Bulgaria, il Montenegro, la Romania, la Serbia ecc. gli elettori all'estero possono votare individualmente. In una lista di altri paesi compresa la Bosnia o la Slovenia la votazione individuale si può eseguire presso le missioni diplomatiche o consolati. Circa 21 paesi votano per posta, compreso il Kosovo, l'Italia, la Slovenia ecc. (KE, 2011).

A seconda dei modelli regionali ed europei esistono anche pratiche di legislazione che creano posti parlamentari riservati per i cittadini che vivono all'estero (Croazia, Francia, Italia, Portogallo, Romania, Macedonia). La scelta tra il modello di quote riservate, la creazione di una zona particolare o la creazione di un sistema di voto unificato nazionale, rimangono pratiche applicate nella regione. Esse testimoniano che non esiste un sistema ideale, però ci sono adattamenti delle specificità di ciascun paese con sistemi e formule ottimali.

\section{Gli Argomenti a Favore della Votazione degli Emigranti}

L'argomento principale a favore della votazione degli emigranti viene costituito dal principio costituzionale della parità e dei diritti, i quali sono obbligatori per tutti i cittadini, dovunque essi abitino. Nel caso albanese la parte occupata dall'emigrazione è talmente alta che l'esclusione dal processo della deliberazione lede sensibilmente anche gli equilibri dell'applicazione del principio costituzionale dei cittadini. L'argomento politico si allarga ulteriormente con il fatto che la loro partecipazione nella votazione influenza positivamente sull'approccio degli emigranti alla vita politica del paese, e l'azione nella politica quale base "flessibile" come citato nel rapporto della Commissione di Venezia.

In riferimento alle pratiche degli altri paesi, compresi i paesi con una popolazione albanese (Kosovo, Macedonia, Croazia, Romania, Serbia, Montenegro), ciascuno di questi paesi ha ormai applicato la votazione della diaspora e l'Albania non può continuare a costituire un'esclusione regionale. La tendenza globale dell'apertura e dell'inclusione richiede inevitabilmente la liberalizzazione del quadro legale della partecipazione attiva dell'emigrazione nei suffragi. A sostegno di tale prassi nel caso albanese vale anche il fatto che l'emigrazione nel corso delle due decadi ha portato un contributo finanziario di $15 \%$ del Pil. Loro oggi non sono più emigranti a livello si sopravvivenza economica, ma hanno cambiato il loro stato sociale, trasformandosi in imprenditori e creando capitali finanziari d'importanza vitale per lo sviluppo della stessa economia albanese. Per una parte della popolazione con un tale contributo sull'economia e sul livello di vita all'interno del paese, non si può ammettere un'esclusione a priori anche dal loro diritto di prendere decisioni sulla politica che deve seguire il paese.

Nel caso albanese esistono anche altri fattori, principalmente culturali e psicologici, che vanno a favore del voto dell'emigrazione. Tale è l'immagine positiva che l'emigrazione ha nella vita pubblica, poiché oramai integrata nelle società occidentali e nel caso di inclusione costituisce un potenziale democratico per la società albanese. L'immagine positiva dell'emigrazione giunge in contrasto con l'immagine negativa della politica albanese, con le delusioni dai partiti tradizionali ed i loro leadership immutabili e accusati di corruzione.

\section{Argomenti Critici nei Confronti del Voto degli Emigranti}

Quando si parla del voto degli emigranti gli argomenti critici si dividono in due direzioni: critiche di principio oppure critiche da parte degli attori politici. Nelle critiche di principio rientra la responsabilità di ogni cittadino di far parte 
dell'ambiente dove si vota e dove si vive la politica e i suoi prodotti. Gli emigranti contribuiscono allo sviluppo del paese, ma questo contributo è principalmente personale e va in linea privata, non in direzione dello stato politico. La maggior parte di loro non sono contribuenti e questo fatto viene considerato spesso un fattore rilevante nel rifiutare il loro ruolo specifico nel processo delle deliberazioni politiche di un paese, per il quale non pagano i contributi. È problematico anche il concetto della classifica dell'emigrante temporaneo, e quello di lungo periodo. Non c'è una netta distinzione, gli stessi emigranti spesso sono indecisi e la loro scelta può essere anche frutto di altre circostanze soggettive ed oggettive e non la loro personale.

Una parte della critica di principio viene costituita dalla problematica tecnica della realizzazione di una potenziale votazione degli emigranti. La prassi delle quote riservate può non essere quella giusta nel caso dell'Albania, ma anche separare gli emigranti in una o due zone elettorali può creare altri problemi relativi all'organizzazione, amministrazione e candidatura. La stessa Albania ha avuto problemi con le elezioni all'interno del paese, perciò esiste una fiducia minima che le ambasciate 0 i consolati all'estero siano istituzioni organizzatori accettabili nelle elezioni politiche. Gli stessi indizi scettici si scontrano anche riguardo agli aspetti del monitoraggio elettorale, la fiducia nel voto preliminare 0 nel voto elettronico, problemi con l'identità degli emigranti ecc. Tra le critiche di principio fa parte anche la titubanza di fronte a scenari negativi p.es. esortazione degli emigranti da qualche paese con pretese politiche in Albania di votare partiti specifici. A causa del grande numero dei voti questa esortazione può essere sufficiente per determinare le sorti del governo e della coalizione vincente a Tirana.

Oltre a questi fattori gli attori politici si accusano a vicenda di essere contrari al voto degli emigranti per ragioni pragmatiche: i partiti in Albania sono molto potenti e continuano a tenere sotto controllo i militanti e le nuove stratificazioni politiche. II voto degli emigranti può diventare una minaccia per il potere assoluto dei partiti, può creare circostanze per una forza o un nuovo movimento politico, può imporre nuove regole del gioco per i quali i partiti locali non sono ancora pronti, nonché può mettere fine ai finanziamenti delle campagne elettorali dei partiti tradizionali. Gli stessi partiti principali preferiscono emigranti che votino a loro favore piuttosto che emigranti che abbiano il diritto di votare anche contro di loro. Per i partiti è più facile commercializzare il voto nell'ambiente povero e tra gli elettori con minimi standard democratici del paese, piuttosto che confrontarsi con un nuovo ambiente, con una nuova cultura di votazione che proviene dai paesi occidentali e così, con un nuovo standard di votazione. Loro sono propensi a considerare gli emigranti come una fonte economica e turistica piuttosto che come attori e voci nella politica.

\section{Conclusioni}

L'Albania è tra i pochi paesi democratici al mondo dove non viene applicata la votazione degli emigranti che vivono all'estero. II dibattito per una soluzione ottimale è all'ordine del giorno, però esiste tuttavia mancanza di chiarezza riguardo alla modalità, efficienza, conseguenze e la stessa volontà degli attori politici locali per rinunciare al sistema monopolio della rappresentanza politica. Tra i numerosi argomenti nel caso albanese c'è posto per la tesi irlandese, secondo la quale, le questioni dell'identità nazionale, del contributo o dell'emigrazione economica non forniscono argomenti solidi per dare il diritto del voto agli emigranti. II maggior argomento si basa sulla necessità di una rappresentanza qualitativa e quantitativa dei cittadini, per i legami continui degli emigranti con il loro paese, nonché per una prospettiva ragionevole del loro ritorno. (Honohan, 2011).

Le soluzioni di breve termine, compresa l'apertura delle filiali dei partiti politici in emigrazione o l'inclusione nelle liste dei candidati provenienti dalla diaspora, sono indizio critico della titubanza per una soluzione sostenibile e a lungo termine. La sfida dell'Albania rimane quella di trovare immediatamente una soluzione ottimale che riunisca le richieste e le potenziali in crescita delle rappresentanze politiche ed economiche della diaspora con le opportunità del sistema attuale politico per una rappresentanza più qualitativa e proporzionale.

\section{References}

Honohan, Iseult. (2011). Should Irish emigrants have votes? External voting in Ireland' Irish Political Studies 26 4: 545-61 (2011)

Geyer, Florian. (2007). Trends in the EU-27 regarding Participation of third-country Nationals in the host country's political life, Brussels, European Parliament, PE. 378.303

Krasniqi, Afrim (2013). Aktiviteti politik i partive jashtë i ligjshëm? www.hermesnews.org/articoli/28683-afrim-krasniqi-partitejashte.asp Meidani, Rexhep. (2010). Beteja për votën (2010), Friedrich-Ebert-Stiftung, 2010

Peter Erben, Ben Goldsmith, Aysha Shujaat. (2012). Out-of-Country Voting: A Brief Overview, IFES White Paper, April.

Qirici, Artjan. (2013). Intervistw, Gazeta Shqip, 2013 (://gazeta-shqip.com/lajme/2013/06/29/vota-emigranteve-atje-ku-jetojne-ps-dokrijohet-qarku-i-13/

Sturm, Roland. (1999). Politische Willensbildung; në: Informationen zur politischen Bildung 262, "Großbritannien", Bonn BpB 
Waldrauch, Harald. (2003). Electoral rights for foreign nationals: a comparative overview of regulations in 36 countries, National Europe Centre Paper No. 73

Documenti strategici

Council of Europe (Venice Commission), Out of Country Voting Legal Provisions, 6.10.2010.

Council of Europe (Venice Commission), Report on Out-of-Country Voting, 24.06.2011.

IDEA, Voting by Mail. The IDEA Technical Paper Series for Election Administrators, 1999.

IDEA, Voting from Abroad - The International IDEA Handbook, 2007. Strategjia Kombëtare për Migracionin, (SKM) 2004

www.dsdc.gov.al/dsdc/pub/national_strategy_for_migration_186_1.pdf

Mbi zbatimin e Planit Kombëtar të Vērimit për Migracionin, Instituti Europian i Tiranës, Tiranë, mars 2007, http://www.soros.al/rmonitorim strategjia emigracioni.pdf

Instituti Shqiptar i Statistikave (INSTAT) [2002]: Regjistri i pergjithshëm i popullsisë për 2001.

Komisioni Europian [2003]: Raporti Vjetor i Statistikave për Migracionin, Azilin dhe Kthimin.

Fletore Zyrtare, Ligji Nr.8580, dt.17.2.2000, "Për partitë politike", 3/2000.

Fletore Zyrtare, 150, nr ligji 9668, Ligji Për emigrimin e shtetasve shqiptarë për motive punësimi", Tiranë 18.1.2007 